\title{
Traffic Sign Detection based on Color Segmentation of Obscure Image Candidates: A Comprehensive Study
}

\author{
${ }^{1}$ Dip Nandi \\ American International University-Bangladesh, Faculty of Science and Information Technology \\ Dhaka, 1212, Bangladesh \\ Email: dip.nandi@aiub.edu \\ ${ }^{2}$ A.F.M. Saifuddin Saif, ${ }^{3}$ Prottoy Paul, ${ }^{4}$ Kazi Md. Zubair, ${ }^{5}$ Seemanta Ahmed Shubho \\ American International University-Bangladesh, Faculty of Science \& Information Technology \\ Dhaka, 1212, Bangladesh \\ Email: ${ }^{2}$ saif@aiub.edu, ${ }^{3}$ prottoypaul634@gmail.com, ${ }^{4}$ kazizubair85@yahoo.com, 5 shubho.aiub@gmail.com
}

Received: 11 April 2018; Accepted: 17 May 2018; Published: 08 June 2018

\begin{abstract}
Automated Vehicular System has become a necessity in the current technological revolution. Real Traffic sign detection and recognition is a vital part of that system that will find roadside traffic signs to warn the automated system or driver beforehand of the physical conditions of roads. Mostly, researchers based on Traffic sign detection face problems such as locating the sign, classifying it and distinguishing one sign from another. The most common approach for locating and detecting traffic signs is the color information extraction method. The accuracy of color information extraction is dependent upon the selection of a proper color space and its capability to be robust enough to provide color analysis data. Techniques ranging from template matching to critical Machine Learning algorithms are used in the recognition process. The main purpose of this research is to give a review based on methods and framework of Traffic Sign Detection and Recognition solution and discuss also the current challenges of the whole solution.
\end{abstract}

Index Terms-Color-based detection, Shape-based detection, Uncontrolled Environment, Multi-class classification.

\section{INTRODUCTION}

Embedded and Intelligent Automated Solution for vehicles for safety in transportation has been the limelight of research in the Computer Vision and Pattern Recognition community for more than thirty years. Pacilik [1] outlined the development of Traffic Sign Detection and Recognition tracking it from its currently popular methods to the paramount study of it in Japan, 1984. The most common automated systems belonging to Traffic Sign Detection and Recognition comprise one or two video cameras mounted on the front of the vehicle (e.g. a geo van). The cars are retrofitted with a PC system for acquiring the videos, or specialized hardware for driving assistance applications Ref. [64]. For convenience Traffic Sign Detection and Recognition will be termed as "TSDR" in this review.

TSDR is a technology that is being researched and enforced within the business industries. Many automobile manufacturers (such as Tesla, Inc., Continental AG) develop the technology to detect and recognize the road traffic signs as a part of the smart vehicle. In 2010, the BMW initiated the project for the production of a TSDR system which was tested by ADAC, the renowned automobile club in Germany Ref. [61]. Volkswagen has also introduced it on the Audi A8 Ref. [62]. Furthermore, Mercedes-Benz has developed traffic sign recognition systems on their E and S class vehicles. Additionally, Google has also started industrialization of self-driving vehicles. Google's autonomous vehicle is able to securely operate in complex urban situations Ref [63].

Road signs have specific properties that distinguish them from other outdoor objects. Operating systems for the automated recognition system of road signs are designed to identify these properties. TSDR usually follows the steps shown in Figure 1. Detection by verification of the hypothesis of the presence of the sign (e.g. equilateral triangles, circles, etc. ). Categorization/Recognition of the type of traffic sign and then detection of the signs from outdoor images is the most complex step in the TSDR system Ref. [2].

Many issues make the problem of the automatic detection of traffic signs difficult such as changeable light conditions which are difficult to control (lighting varies according to the time of the day, season, cloud cover and other weather conditions) Ref. [14]. Presence of other objects on the road (traffic signs are often surrounded by other objects producing partial occlusions, shadows, etc.) also hinders the detection process Ref. [54-56].

The research takes a precarious turn when considering the possibilities that can cause false positives since the 
algorithm has to take camera distance and view angle of the recorded image along with possible deformation caused by external factors to the signs' appearance into account.

Hence, any robust TSDR solution must provide straightforward results that are not affected by perspective distortion, lighting changes, partial occlusions or shadows [3]. Ideally, the solution should also provide additional information on the lack of visibility, poor conditions and poor placement of traffic signs. In Section III, the methods applied in various TSDR solutions are going to be discussed. An in-depth analysis of the proposed frameworks for TSDR and the experimental results of some frameworks are going to discussed in Section IV and IV respectively

\section{ANALysis Aspects OF TrafFic Sign Detection AND RECOGNITION}

Generally, TSDR can be divided into four phases: Video/Image/Frame Acquisition, Preprocessing, Detection and Recognition. Among them, Detection and Recognition is the most crucial part of the solution

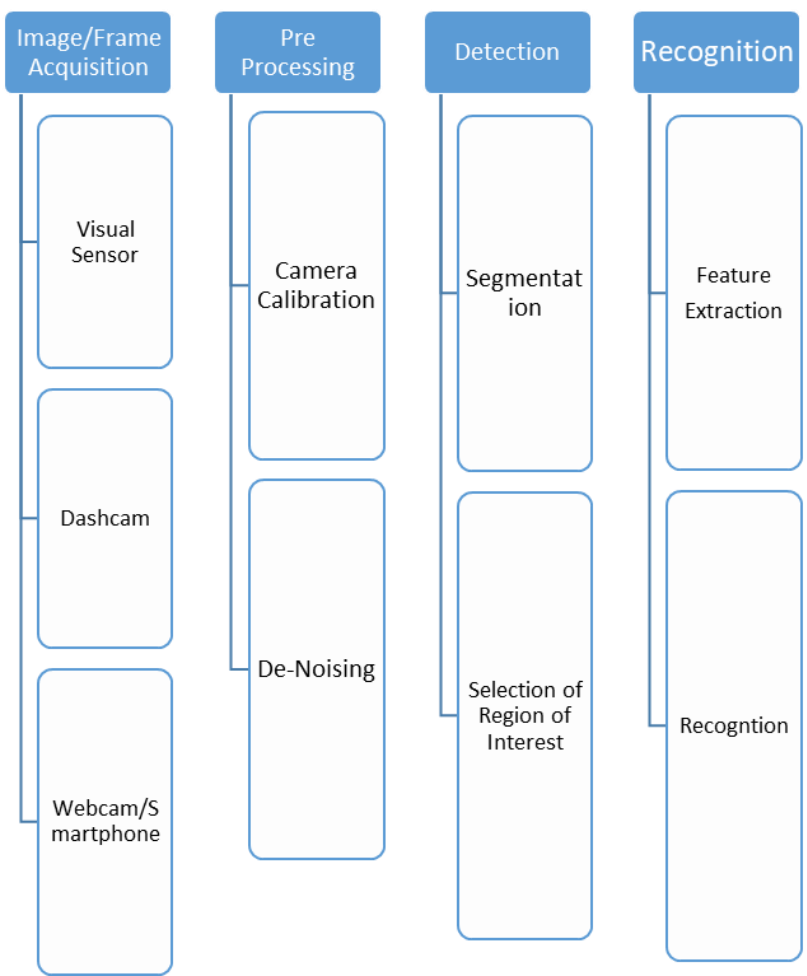

Fig.1. General framework followed in Traffic Sign Detection and Recognition

In detection phase Segmentation is one of the most important steps as it helps to extract ROI from the sample. Segmentation is generally divided into two categories: Color-based approaches and Shape-based approaches. In most color segmentation cases color thresholding is used. When using color information in road sign detection methods, change in lighting, strong illumination and adverse weather conditions can result in problematic changes. These problems were overcome by using Color models such as HSV [14], [9], [11], [25], [26], YUV [13], YCbCr [18, 38, 40] and CIECAM97 [15]. Although color based approaches produce faster results in most of the cases it reduces the detection rate in the cluttered environment. In shape-based approaches, Hough transformation, Radial Symmetry, Template matching is the most popular [60]. A novel ROI extraction method, called High Contrast Extraction in combination with occlusion robust recognition method based on Extended Sparse Presentation Classification (ESRC) was introduced in the recognition process in Ref. [20]. The most widely used supervised learning approaches for traffic sign recognition are ANN Ref. [6, 25, 26, 28, 36, 38, 40],SVM Ref. [12, 21, 23], Random Forest Ref. [33, 34, 37, 42] etc. AdaBoost Ref. [7, 24, 42] is also a prominent candidate in TSDR solutions. Soetedjo \& Yamada Ref. [44, 45] and Kobayashi [41] used GA for TSDR solution.

All TSDR generally follows the framework described in Figure 1. One of the currently followed frameworks is deep learning based framework such as Convolutional Neural Network (CNN) Ref.[29, 30, 31].CNN pioneered the way parallel programming with high-end GPU which provides high efficiency in real time application Ref.[29].In the field of Bengali based TSDR progress is only little. The existing state of earth frameworks is not feasible for real-time implementation.

From experimental results, we can see that $\mathrm{CNN}$ produce a much greater result if the datasets increases which result in need of memory spaces. Shortage of public dataset is also a huge problem, for this reason, all TSDR are limited to only certain country's traffic sign.

\section{REVIEW BASED ON METHODS}

A comprehensive review of the common methods used in TSDR solutions as represented in the image below is the scope of this section.

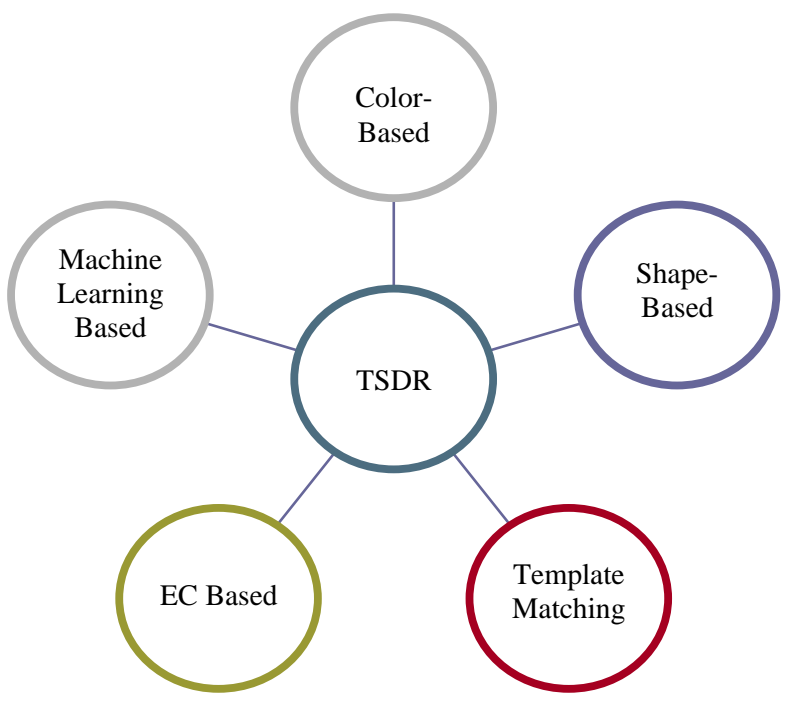

Fig.2. Methods of Traffic Sign Detection \& Recognition 
Traffic Sign detection using color is based on the five typical colors defined in standard traffic signs (red, blue, yellow, white and black). Robust color segmentation especially considering non-homogeneous illumination is given priority, since errors in segmentation may be propagated in the following steps of the system.

Color is usually perceived as a combination of three primary colors: Red, Green, Blue is also known as RGB. From RGB other color transformations such as HSV, YUV and CIECAM97 can be extrapolated using linear and non-linear transformation Ref. [58]. RGB is the most commonly used method for any type of display system and photos acquired by digital camera Ref. [59]. The drawbacks of using RGB color space in segmentation includes the complexities caused by the $3 \mathrm{D}$ nature of the system and the high correlation present between color components. The latter results in the variation of ambient light intensity, affecting the RGB components by shifting the cluster of colors towards the white or the black corners. Therefore segmenting an object using RGB space using the same set of thresholds will not be possible under varying lighting conditions.

To reduce intensity dependency another color space has been introduced known as HSV. HSV stands for Hue, Saturation, and Value. Since the HSV color space is less sensitive to variable lighting conditions, many researchers considered using this method for traffic sign segmentation. For instance, Paclik et al. Ref. [1] used this color space due to its similarity to human perception of colors. The hue channel of HSV color space was thresholded by Malik et al. Ref. [9] for color segmentation of red traffic signs. Segmentation was done by Shadeed et al. Ref. [13] by implementing the U and V chrominance channels of YUV space where "U" is regarded as positive and " $\mathrm{V}$ " as negative for red colors. The hue channel of HSV color space, in combination with YUV space information, was used to segment red colored traffic signs. Gao Et Al. Ref. [15] applied a quadtree histogram method to segment the image based on the hue and chroma values of the CIECAM97 color model. The CIECAM97 color model consisting of hue and chroma values were used by Ref. [15] to segment images implementing the quad-tree histogram method. Recently in digital images and video streams, another color space named $\mathrm{YCbCr}$ is frequently used. Y represents the luminance component which indicates the light intensity of the color. $\mathrm{Cr}$ and $\mathrm{Cb}$ are the chrominance components of which $\mathrm{Cb}$ is the blue and $\mathrm{Cr}$ is the red component relative to the green component respectively. These components are less sensitive to the human eyes. Dean and Jabir in [40] used RGB to YCbCr o thresholding based on red and blue color of the traffic sign.

Detection of Traffic Signs via its shape follows the defining algorithm of shape detection i.e. to finding the contours and approximating it to reach a final decision based on the number of contours. One of the key advantages of shape-based detection is that any variation in daylight or colors does not affect it. Generally, Traffic Signs come in circular, triangular, rectangular, octagonal etc. shapes. Usually, after image preprocessing, the shape-based detection is done. Hough transformation is one of the most commonly used among shape detectors. Hough Transformation usually isolates features of a particular shape within a given frame/images. Classic Hough Transformation is concerned with the identification of regular curves such as a circle, rectangle. Later Hough Transformation is customized to detect complex curves. García-Garrido, Sotelo \& MartmGorostiza in Ref. [47] used Hough Transformation to detect circular and triangular signs. Also Kuo \& Lin in Ref. [48] combined Hough Transformation with an iterative process of median filtering and dilation to refine the sign candidate set. One of the major advantages of Hough transformation is that it is unaffected by noise and illumination variance. But in case of high-quality video frames, Hough Transformation's computational cost becomes expensive which makes it improper for RealTime implementation. To overcome this computational expense Radial Symmetry method was introduced. The failure rate of this method increases with the increase of edges. Loy \& Zelinksy Ref. [17] theorized a method to highlight points of interest that detect octagonal, square and triangular traffic signs using local radial symmetry. But shape-based segmentation has many disadvantages such as occluded, damaged, disoriented, improper viewing angle and varying aspect ratios. But some difficulties do occur when using shaped based detection. Notable shape-based detection problems of TSDR solutions are discussed below.

Traffic signs are mostly designed and constructed using basic shapes like circles, triangles, pentagons etc. to make it easily visible. But similarly shaped objects also exist in the surroundings that are not traffic signs. Traffic signs are prone to physical damage and being obstructed from view. The size of traffic signs compared to its real size depends on factors such as the distance between the camera lens and traffic sign. The camera view might also be disoriented vertically or horizontally. Moreover, factors like small object size of a traffic sign in images and slanted angle of view create difficulties in the detection phase due to change in aspect ratio. Small roughly distinguishable traffic signs in images make it rather difficult to approximate contours and so robust edge detection and recognition algorithms are necessary.

In order to overcome the problems of color based and shape based detection, some hybrid methods have been proposed. Color segmentation followed by shape analysis is usually done in these hybrid methods Oruklu et al. Ref. [50]. At first, the color segmentation is done in the HSV color space and then boundary boxes are inserted for all the regions detected through the color segmentation. The traffic sign is detected by using the features of the bounding boxes such as mean color, size, and a number of pixels enclosed in the boundary box. Zheng et al Ref. [51] used both the color and shape information to detect the traffic signs. The image is color segmented by using RGB color space and then shape analysis is performed by using the Douglass-Peucker algorithm which is a contour approximation technique and the detection is based on the 
number of object boundaries which enables it to detect signs despite having some geometric distortions.

TSDR uses Template Matching based algorithms that search for existing similar training samples which are then stored in a database as unknown Regions of Interest (ROIs). Template matching is performed on these ROIs to recognize traffic signs. A robust method was mentioned in Ref. [60] where Template matching was performed for simultaneous detection and recognition. The input was compared with differently shaped sign templates extracted from various ROIs and then were grouped together under a tree structure based on their similarity.

Machine Learning methods in the Traffic Sign Detection and Recognition are the most commonly used. Machine Learning methods can be categorized into three types: Supervised Learning, Unsupervised Learning, and Reinforcement based learning as shown in Figure 2 Ref.[65].

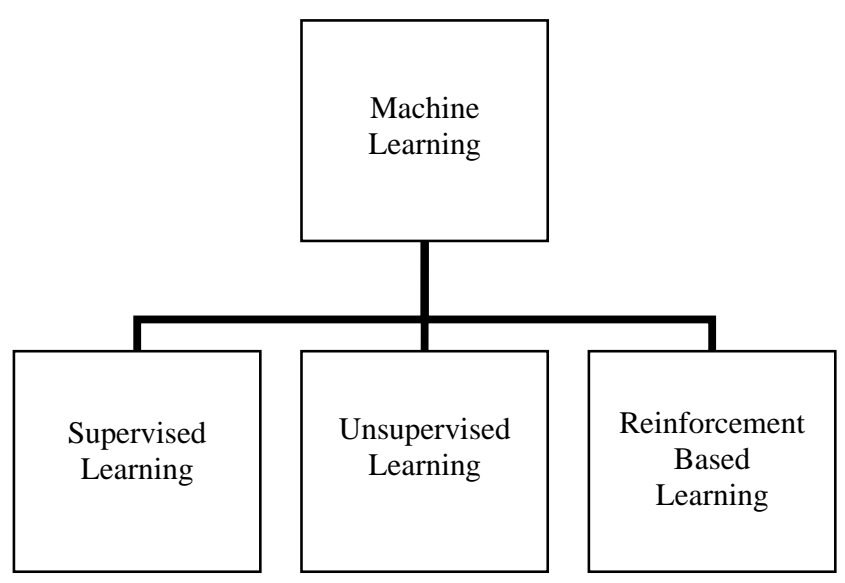

Fig.3. Machine Learning Paradigms

Among the machine learning methods supervised learning methods are the most conventional in the proposed TSDR systems. Supervised learning methods build/learn a model from given datasets then predicts a response to future instances Ref. [52]. Classifier models are created to train feature vectors that can distinguish between different traffic signs in supervised learning paradigm.

Support Vector Machine (SVM) is a supervised machine learning method which classifies points to one of two disjoint half-spaces Ref.[68]. Authors in Ref. [35] uses multilevel SVM to overcome the binary classification. Multi-level SVM is used to recognize different shapes of signs. Ref.[36] and Ref.[39] used linear SVM for recognition purpose. SVM's advantage is: it's performance increase while working with non-linear boundary based on a kernel and it works well with high dimensional data. But SVM is prone to overfitting which is a major flaw.

Artificial Neural Network is recently being used in TSDR solutions. ANN gives a major advantage in case of Real Time TSDR because of its fast response and computational time. But during overfitting reduction, it increases computational time. Among ANN multi-layer perceptron (MLP) is most frequently used in TSDR. A multi-layer perceptron, also referred to as MLP, is a feedforward artificial neural network and it consists of multiple layers, which are fully connected to each other Ref.[71]. In other words, every single neuron in a multilayer perceptron is linked to the neurons in the next layer. The advantages of Multi-layer Perceptron are Capability to learn non-linear models. Capability to learn models in real-time (online learning) using partial fit. The disadvantages of Multi-layer Perceptron (MLP) is it requires tuning a number of hyperparameters such as the number of hidden neurons, layers, and iterations and sensitive to feature scaling. In [25] A road sign image which contains $50 \times 50$ pixels is proceed to classification sub-module, and Multi-Layer Perceptron (MLP) network [4] has been created and trained to classify the type of road signs in this sub-module. The input signal transmits through the network in a forward path, on a layer-bylayer basis. The network has been trained on 300 sets of training patterns taking 75 for each type of road sign and 200 test patterns taking 50 images of each road sign. The network has 7500 input neurons and 4 output neurons. The hidden layer is 5. Authors in Ref. [26, 28, 38, 36] MLP is used in recognition process.

Recently Convolutional Neural Network has become a very popular method in the field of real-time Automated Driving Assistance System. One of the cons of CNN is a large number of datasets which increases training time. But it is also an advantage as the large the dataset the more accurate is the result. Also increase in layer means more features are added to training set which allows CNN to work with high-resolution images. Also, CNN possess complicates structures.

In Ref.[30] combination of two architectures (AlexNet AND GoogLeNet) of CNN has been used. Authors in [31] used Faster Region based CNN(R-CNN). The Faster RCNN network [49] is composed of two modules: Region Proposal Network (RPN) and Fast R-CNN.

RPN produces a set of bounding box object proposals of the input image which Fast R-CNN classifies into the set of predefined categories. Fast R-CNN is highly efficient since it shares convolutions across individual proposals. It also performs bounding box regression to further refine the quality of the proposed regions.

Decision Tree is a supervised learning model for classification and regression. Decision trees are favored because it doesn't require any domain knowledge, parameter setting and can handle multidimensional data with fast speed and good accuracy. Among Decision Tree methods Random Forest is used in most proposed solution for traffic sign recognition. The main reason behind using Random Forest is the ability for multi-class detection in contrast to common boosting detectors A Random Forest consists of several independent decision trees arranged in a forest. A majority vote over all trees leads to the final decision. Random forests are trained in a supervised way. Authors in [42] used an Error Correcting Output Code also known as ECOC framework, on the Random forest which increases the efficiency. 
Adaptive Boosting or AdaBoost is a combination of multiple learning algorithms that can be utilized for regression or classification [72]. In Ref. [24] AdaBoost is used on static images in a different environment to detect text based recognition. In [42] an evolutive Adaboost method has been introduced for illumination invariance and to enhance the recognition rate.

Evolutionary techniques mostly involve meta-heuristic optimization algorithms. The basic evolutionary algorithms (EA) encompass genetic algorithm, genetic programming, and evolution strategies. EA shares the common theme of optimization performed on a population of potential solutions applying techniques inspired by biological evolution to produce better approximations to a solution. Genetic Algorithm is the most used technique of Evolutionary Computation, it is a fast and accurate algorithm which can outperform Neural Networks and SVM in some particular tasks. It is therefore very useful in TSDR solutions.

\section{REVIEW BASED ON FRAMEWORKS}

Most of the TSDR solutions usually follow the framework mentioned in Figure 1. Based on this general framework up to now many frameworks have been proposed and implemented. In this section, some of these existing frameworks from Table 1 are going to be discussed along with their advantages and some possible issues.

In Ref. [25] it was observed that salt and pepper noise reduction in pre-processing, HSV color based segmentation in detection part and Multi-Layer Perceptron (MLP) in recognition phase were used respectively. Even though conversion to $\mathrm{HSV}$, noise reduction etc. in pre-processing minimalizes error, there can be a change in physical color of the object i.e. Traffic Sign in reality which will affect Color Segmentation after detection to HSV.

Ref [26] uses fuzzy logic on color based segmentation, SURF descriptor for feature extraction and MLP for recognition. For using SURF descriptor, Traffic signs are detected and classified after extraction based on fuzzy rules segmentation which provides robustness and also gives a very high accuracy for static detection and recognition. But lack of pre-processing and only using fuzzy techniques with logical AND based results may cause some errors. In Ref. [28] a hybrid method utilizing the combination of RGB color space with shape and size constraint analysis based segmentation is used in detection phase along with auto-associative neural network for sign identification. This framework produces highly accurate results only in static images. But in case of real-time implementation with the change of orientation, weather conditions, lighting and also the speed of the vehicle will decrease its accuracy.

The Framework in Ref. [29] uses multiple HSV color space thresholding with Hough transformation algorithm to detect traffic signs and then uses LeNet-5 Model, a deep convolutional algorithm to recognize the extracted ROIs. It is inefficient for a real-time solution due to its parallel programming through high powered GPU. As for using two separate segmentation on the same frame, overlapping of ROIs produce a redundant result and also increases computational cost. The accuracy is dependent solely on weather and illumination conditions along with the number of datasets used to train the algorithm. Also as LeNet-5 is constrained by computational resources it is not available for higher resolution frames. In case of Ref. [30] a framework using HIS combined with selective search algorithm in detection phase and also AlexNet and GoogLeNet, two convolutional Neural Network Algorithm for recognition was theorized. Selective Search is used mainly for its ability to process at a high speed during an exhausting search along with the robustness it provides for distinguishing objects based on size, color, texture and region similarity. AlexNet and GoogLeNet convolution network are used for attaining accuracy on recognition. Uršič et al. Ref. [31] used data augmented enriched dataset and Fast R-CNN Ref. [49] for both detection and recognition phase. Region-Based Convolutional Neural Networks(R-CNN) and Region Proposal Network Ref. [49] are highly efficient since they share convolutions across individual proposals. They also perform bounding box regression to further enhance the quality of the proposed regions. It enables fast and rapid detection of TSDR solutions. Even though it is very fast it is a three-stage training phase for R-CNN and it requires a large amount of space along with sufficient GPU power. Also in some cases, the overlapping of ROIs increase the computational time.

Ellahyani, Ansari, Jaafari, and Charfi in Ref. [34] proposed a framework which uses a hybrid method in detection combining HSI color thresholding with DtBs shape analysis in detection phase and multiple feature extraction methods (HOG \& LSS) with the random forest as a classifier. In this framework, DtBs shape analysis method is invariant to scaling, translation, and rotation respectively due to the normalization of the DtBs vectors to the bounding-box dimensions. The position of candidate blob does not matter and the most extreme pixels of each blob is detected to determine the original orientation. All blobs are then oriented in a reference position. Random Forest classifier is robust and tolerant to noise compared to other classifiers. But the main drawback of this method is basic thresholding which is not invariant to weather changes. Although the framework produces a more accurate result, the computational time it requires is high. 
Table 1. Existing and Proposed Frameworks for Traffic Sign Detection and Recognition Solution

\begin{tabular}{|c|c|c|c|}
\hline Major Steps Used & Ref. & Advantages & Possible Issues/Disadvantages \\
\hline $\begin{array}{l}\text { Color Feature and Multilayer } \\
\text { Perceptron Neural Network }\end{array}$ & [25] & - Less reactive to illumination changes. & $\begin{array}{l}\text { - Lacks robustness in terms of illumination } \\
\text { changes and faded signs. } \\
\text { - Failure in multiple sign detection }\end{array}$ \\
\hline $\begin{array}{l}\text { Fuzzy Color Segmentation, } \\
\text { Filtering, Speeded Up Robust } \\
\text { Features(SURF) Extraction, } \\
\text { Multilayer Perceptron Neural } \\
\text { Network } \\
\end{array}$ & [26] & $\begin{array}{l}\text { - High accuracy in the recognition process. } \\
\text { - Fuzzy segmentation is robust. }\end{array}$ & $\begin{array}{l}\text { - Lack of pre-processing } \\
\text { - Using fuzzy techniques with logical AND } \\
\text { based results may cause some errors. }\end{array}$ \\
\hline $\begin{array}{lr}\text { Shape \&Size } & \text { Constraints, } \\
\text { Auto-Associative } & \text { Neural } \\
\text { Network } & \\
\end{array}$ & [28] & $\begin{array}{l}\text { - Highly accurate recognition rate in static } \\
\text { images }\end{array}$ & - Accuracy reduced in a shadowed environment. \\
\hline $\begin{array}{ll}\text { Hybrid } & \text { Method(HSV } \\
\text { segmentation } & + \text { Hough } \\
\text { Transformation), LeNet } & -5 \\
\text { Model } & \end{array}$ & [29] & $\begin{array}{l}\text { - Parallel Programming for high powered } \\
\text { GPUs enables the validation of signs in } \\
\text { real time efficiently. }\end{array}$ & $\begin{array}{l}\text { - Produce Redundant results } \\
\text { - High computation cost at the detection } \\
\text { - Does not produce good results in the high- } \\
\text { resolution camera } \\
\text { - Vulnerable to illumination changes } \\
\end{array}$ \\
\hline $\begin{array}{lr}\text { Selective Search } & \text { Detection, } \\
\text { Convolutional Neural Network } \\
\text { Learning, } \\
\text { Recognition(CNN) }\end{array}$ & {$[30]$} & - Faster Region of Interest extraction. & $\begin{array}{l}\text { - Reflective light and back-light will cause the } \\
\text { detection failure. } \\
\text { - Too many ROI's efficiencies of CNN } \\
\text { algorithm. }\end{array}$ \\
\hline $\begin{array}{l}\text { Data Augmentation, Faster } \\
\text { Region Based Convolutional } \\
\text { Neural Networks (R-CNN) }\end{array}$ & [31] & $\begin{array}{l}\text { - Enriched Dataset } \\
\text { - Fast computation. } \\
\text { - Illumination Invariants }\end{array}$ & $\begin{array}{l}\text { - Overlapped ROI's increases computational } \\
\text { time. } \\
\text { - Failure to detect some undistorted signs }\end{array}$ \\
\hline $\begin{array}{l}\text { Coarse classification, Fine } \\
\text { classification- Dense-SIFT, } \\
\text { LBP, Gabor filter fusion, a } \\
\text { fusion of SVM and Random } \\
\text { Forests(RF) }\end{array}$ & [33] & $\begin{array}{l}\text { - Fusion of different methods increases the } \\
\text { rate of success. }\end{array}$ & $\begin{array}{l}\text { - Low resolution and bad illumination situations } \\
\text { along with the specific disadvantages of the } \\
\text { different classifiers and feature extractors. }\end{array}$ \\
\hline $\begin{array}{l}\text { HSI color thresholding, } \\
\text { Distance to Borders (DtBs), } \\
\text { HOG, LSS, Random Forest. }\end{array}$ & [34] & $\begin{array}{l}\text { - Invariant to translation, scale, and rotation } \\
\text { - Accurate and Robust to noise }\end{array}$ & $\begin{array}{l}\text { - Weather condition effects on the whole } \\
\text { process. } \\
\text { - Huge computational time }\end{array}$ \\
\hline $\begin{array}{l}8 \text { bit Modified } \text { Census } \\
\text { Transform, } 4 \text { stage based } \\
\text { cascade AdaBoost classifier, } \\
\text { Multilevel SVM }\end{array}$ & [35] & $\begin{array}{l}\text { - } \text { Illumination invariant } \\
\text { - Robustness against occlusion and } \\
\text { distortion } \\
\text { - } 3 . \text { The less Computational cost in } \\
\text { recognition. } \\
\end{array}$ & - Less accuracy compared to other frameworks. \\
\hline $\begin{array}{l}\text { Bilateral Chinese Transform } \\
\text { (BCT), Vertex and Bisector } \\
\text { Transform (VBT), HOG } \\
\text { feature, Linear SVM, Multi- } \\
\text { Layer Perceptron }\end{array}$ & [36] & - BCT and VBT reduce the ROI. & $\begin{array}{l}\text { - Computational Cost is high. } \\
\text { - Limited to circular and triangular sign. }\end{array}$ \\
\hline $\begin{array}{l}\text { Color enhancement (red), } \\
\text { Chromic filter, Morphological } \\
\text { filter. HOG Featured SVM, } \\
\text { Random forest classification }\end{array}$ & [37] & $\begin{array}{l}\text { - Reduces the search space and accelerates } \\
\text { the detection. } \\
\text { - Faster performance in an embedded } \\
\text { system. }\end{array}$ & - Performance reduces with smaller training sets. \\
\hline $\begin{array}{l}\text { Morphological Classification. } \\
\text { Principle Component Analysis } \\
\text { with MLP. }\end{array}$ & [38] & $\begin{array}{l}\text { Simple and Faster implementation with } \\
\text { higher accuracy }\end{array}$ & $\begin{array}{l}\text { - Failure to recognize damaged, occluded } \\
\text { signs }\end{array}$ \\
\hline $\begin{array}{l}\text { Fast Fourier Transformation. } \\
\text { Normalization of ROI, HOG } \\
\text { based extraction, Linear SVM }\end{array}$ & [39] & - Reduces computational time of detection. & $\begin{array}{l}\text { - High computational time. } \\
\text { - Sensitive to illumination. }\end{array}$ \\
\hline $\begin{array}{l}\text { YCbCr Color segmentation, } \\
\text { shape filtering based on } \\
\text { template matching, Multi-layer } \\
\text { Perceptron Neural Networks }\end{array}$ & [40] & - Faster Detection. & $\begin{array}{l}\text { - Shape and color based detection rate can be } \\
\text { reduced due to occlusion and distortion. }\end{array}$ \\
\hline $\begin{array}{l}\text { HSV color segmentation, } \\
\text { Hough } \text { Transformation, } \\
\text { Genetic algorithm based } \\
\text { Template matching }\end{array}$ & [41] & $\begin{array}{l}\text { - Produces good result in adverse conditions } \\
\text { such as shadowed, occluded. } \\
\text { - No dataset }\end{array}$ & $\begin{array}{l}\text { - Static image implementation. } \\
\text { - Spurious object detected. }\end{array}$ \\
\hline $\begin{array}{ll}\text { Evolutive AdaBoost, } & \text { Forest- } \\
\text { Error-Correcting } & \text { Output } \\
\text { Codes(ECOC) } & \\
\end{array}$ & [42] & $\begin{array}{l}\text { - Speeds up the learning process } \\
\text { - Allows working with a large feature. }\end{array}$ & - Requires huge computational time. \\
\hline
\end{tabular}


Table 2. Experimental Result of Proposed methods for traffic sign detection and recognition

\begin{tabular}{|c|c|c|c|c|}
\hline Reference & Dataset & Sign Shapes & $\begin{array}{l}\text { Experimental } \\
\text { Environments }\end{array}$ & Numerical Estimation \\
\hline [25] & & Circle, Triangle Hexagonal & Sunny & $\begin{array}{l}\text { Detection rate }=90.9 \% \text { and the accuracy of Recognition } \\
=88 \%\end{array}$ \\
\hline [26] & $\begin{array}{c}\text { Syntactically } \\
\text { Created }\end{array}$ & $\begin{array}{l}\text { Circle, Triangle } \\
\text { Quadrangular }\end{array}$ & Sunny & Detection Rate $=95 \%$ and Recognition Rate $=97 \%$ \\
\hline [28] & & $\begin{array}{l}\text { Circle, Triangle } \\
\text { Hexagonal, Rectangle }\end{array}$ & Daylight, Shadow & $\begin{array}{l}\text { Color Segmentation Accuracy }=93.3 \% \text { in Daylight and } \\
95 \% \text { in Shadow respectively. } \\
\text { Recognition Rate in Daylight } 100 \% \text {, Shadow } 94.7 \%\end{array}$ \\
\hline [29] & Korean TSD & Circle, Triangle Rectangle & Daylight, Shadow & $\begin{array}{l}\text { Training with only Positive samples i.e. real signs and } \\
\text { after that mixing training data with } 25,000 \text { samples of } \\
\text { real signs and } 78,000 \text { false positives }\end{array}$ \\
\hline [30] & GTSDB & Circle, Triangle Rectangle & Daylight & $\begin{array}{l}\text { Detection Rate }=92.63 \% \text { and Recognition Rate }= \\
80.5 \% \text {, }\end{array}$ \\
\hline [31] & $\begin{array}{l}\text { DFG } \\
\text { Consulting } \\
\text { d.o.o. company } \\
\text { Dataset }\end{array}$ & Circle, Triangle Rectangle & & $\begin{array}{l}\text { With augmented dataset rate of success }=90 \%, \\
\text { augmented training set using images with synthetically } \\
\text { generated templates }=6388 \text { images, the rate of success }= \\
88.5 \%\end{array}$ \\
\hline [32] & BTSD & $\begin{array}{l}\text { Circle, Triangle } \\
\text { Hexagonal, Rectangle }\end{array}$ & & Detection Rate $91.69 \%, \quad$ Recognition Rate $=93.77 \%$ \\
\hline [33] & GTSRBD & Circle, Triangle Rectangle & & Recognition Rate $=98.76 \%$ \\
\hline [34] & STSD & Circle, Triangle, Rectangle & & Recognition Rate $=96.13 \%$ \\
\hline [35] & $\begin{array}{l}\text { KOREAN } \\
\text { TSD+GTSDB+ } \\
\text { DTSRBD }\end{array}$ & Circle, Triangle Rectangle & $\begin{array}{l}\text { Daytime, Rainy, } \\
\text { Shadow, Foggy }\end{array}$ & $\begin{array}{l}\text { Recall }=99.6 \% \\
\text { Precision }=99.0 \%\end{array}$ \\
\hline [36] & & Circle, Triangle Rectangle & & $\begin{array}{l}\text { Recall }=90 \% \\
\text { Precision }=88 \%\end{array}$ \\
\hline [37] & & Circle, Triangle Rectangle & & $\begin{array}{l}\text { Recall }=90.21 \% \\
\text { Precision }=90.90 \% \\
\text { Recognition Rate }=97 \%\end{array}$ \\
\hline [38] & & Circle, Triangle, Rectangle & & $\begin{array}{l}\text { Recognition Rate: } \\
\text { Circle }=96 \% \\
\text { Square }=91 \% \\
\text { Triangle }=95 \% \\
\end{array}$ \\
\hline [39] & & Circle, Triangle, Rectangle & & Accuracy rate $=95.5 \%$ \\
\hline [40] & & Circle, Triangle Rectangle & & The correct recognition rate $=90 \%$. \\
\hline [41] & & Circle, Triangle Rectangle & Daytime, Night & Recognition rate $=94.9 \%$ \\
\hline [42] & UCI dataset & Circle, Triangle Rectangle & & Accuracy Rate $=90 \%$ \\
\hline
\end{tabular}

Census Transform and Multilevel Support Vector Machine Ref. [35] method displays high illuminationinvariant accuracy in detection and recognition but in case of urban areas, its accuracy decreases. Shi and Lin in Ref. [36] used HOG and SVM in the first step, and Bilateral Chinese Transform (BCT), Vertex and Bisector Transform (VBT) and color information to determine the exact sign region. Then the sign regions are normalized to a size identical to the training samples and classified by the backpropagation based neural network. Bilateral Chinese Transform (BCT), Vertex and Bisector Transform (VBT) reduce the ROI but their accuracy rate declines considerably.

Thanh in Ref. [38] proposed a framework which uses Morphological classification for ROI, AND Principle Component Analysis (PCA) and Multi-Layer Perception Network (MLP) in recognition. Although Morphological classifications are fast, they are disadvantageous because they cannot detect damaged signs.

Baro et al. in Ref. [42] used Evolutive AdaBoost in detection process and Forest-ECOC for recognition. Evolutive AdaBoost speeds up the learning process with huge sets of features. The whole framework is designed in such a way to produce high accuracy and robustness against a high variability of sign appearance.

Rahman et al at [70] proposed a system in which structured in a sequence of processes such as Image Acquisition, Preprocessing, Text Detection and Extraction, Bengali Optical Character Recognition, Confirmation of Textual Road Sign, and Conversion to Audio Stream using Speech Synthesis. In the detection step the extracted edge based on Sobel operator then color segment to reduce ROI. Then Text containing ROI is extracted. Then MLP is used for optical character recognition for Bengali words. Although it managed to detect Bengali text signs it is highly inefficient in case of illumination changes, occluded signs etc. Another major problem is as it is text segmented if there are other signs containing an advertisement or else it will detect them as a sign which leads to redundant data.

Chakraborty and Deb in [18] used $\mathrm{YCbCr}$ Segmentation with Distance to Border Shape analysis in detection stage on Triangle, Quadrangular, and Circular signs. 


\section{REVIEW BASED ON EXPERIMENTAL RESULTS}

This section is an extensive review of the experimental results of the frameworks discussed in Section IV. Color Feature and Multilayer Perceptron Neural Network in Ref. [25] can detect circle, triangle and hexagonal shapes in a sunny environment with detection rates of $90 \%$ and an $88 \%$ accuracy in recognition rate. Fuzzy Color Segmentation, Filtering, Speeded Up Robust Features (SURF) Extraction, Multilayer Perceptron Neural Network are used in Ref. [26]. Using syntactically generated datasets, most shapes including quadrangular can be detected in sunny conditions at an accuracy of 95\% and recognized at a rate of $97 \%$ accuracy.

A detection rate of $93.3 \%$ in daylight and $95 \%$ in shadow for color segmentation with a recognition rate of $100 \%$ and $97 \%$ in daylight and shadow respectively was observed in Ref. [28] where Shape Size Constraints and Auto-Associative Neural Networks were the methods used. Korean TSD is a dataset where training was done with only Positive samples i.e. real signs and after that mixing training data with 25,000 samples of real signs and 78,000 false positives in the implementation of Ref. [29] where Hybrid Method (HSV segmentation + Hough Transformation) and LeNet-5 Model were used. In Ref. [30] Selective Search Detection, Convolutional Neural Network Learning, and Recognition (CNN) were used with the GTSDB dataset with a detection rate of $92.63 \%$ and recognition rate $80.5 \%$ in sunny conditions.

Mostly circular, triangular and rectangular objects were detected in Ref. [31] where the use of Data Augmentation, Faster Region Based Convolutional Neural Networks (RCNN) was observed. The augmented dataset rate of success was $90 \%, 6388$ images obtained by synthetically generating templates were used as an augmented training set. The rate of success was $88.5 \%$.

Coarse classification, Fine classification-Dense-SIFT, LBP, Gabor filter fusion, a fusion of SVM and Random Forests (RF) was part of the framework in Ref. [33] where the GTSRBD dataset was used to obtain a result with the recognition rate of $98.76 \%$. Similarly, Ref. [34] was another solution where Random Forests Classifier was used and a recognition rate of $96.13 \%$ was observed with the STSD dataset.

8 bit Modified Census Transform, 4 stage based cascade AdaBoost classifier, Multilevel SVM were used in implementing a hybrid method with the combined dataset of KOREAN TSD, GTSDB and DTSRBD to attain a result of $99.6 \%$ recall and $99 \%$ precision Ref. [35]. Similar SVM based methods with Bilateral Chinese Transform, Multi-Layer Perception, and Morphological operations as seen in Ref. [36, 37, 38] with recognition rates of $88 \%, 97 \%$ and $96 \%$ respectively. In Ref. [39] Linear SVM was the primary method of classification with test 9171 test datasets and an accuracy of $95.5 \%$.

Ref. [40] and Ref. [41] mostly based on Multi-layer Perceptron Neural Networks and Template matching based on Genetic Algorithm methods managed to obtain $94.9 \%$ and $90 \%$ accuracy rate respectively.
Finally in Ref. [42] an Evolutive Adaboost method was used to attain an accuracy rate of $90 \%$ for most circular, triangular and rectangular shapes.

\section{OBSERVATION}

The environment for Traffic Sign Detection and Recognition system is an adverse one with many unaccounted variables mostly physical. Some of the problems and difficulties while the system is active and working are discussed below:

1. From the above-discussed review, it is very apparent that there is a small number of proposed frameworks for night time. Also, most of the existing frameworks' accuracy drops as it is not easy to perceive the traffic sign without a high-resolution camera. The difficulty arises due to low contrast at night. Also due to reflective plates of traffic signs remain undetected in color thresholding based.

2. A video captured from a moving vehicle often causes a problem name motion blur. Due to motion blur, sharp edges of the candidate object especially the frameworks with shape analysis based algorithm suffers from it. Thus, to restore a motion blurred image a restoration algorithm is required. Although there is some restoration algorithm is available it increases the computational time which is a hindrance in Real Time application like Driving Assisting System. Sometimes Traffic sign became distorted due to an unsuitable viewing angle of the camera for which an algorithm to reconstruct or fit the shape in the image plane is required. In state-of-the-art Traffic Sign Detection and Recognition, the shape of the traffic sign is spotted by using appearances of the shape and existence of candidate is detected by segmentation. Often to reduce the error due to the detection of non-road sign object, a fusion of color information with features of candidate signs is proposed to generate different layered structures among different classes.

3. From above sections, we can see that there are hardly any methods or frameworks that worked with detection and recognition of heavy rain or foggy weather. Especially those with shape-based detection algorithm suffers as foggy weather hides all distant objects from the camera. Due to rain droplets, edges of traffic signs gets hard to detect. Haze also affects all the edges of the image. Although there are algorithms to identify rain or fog automatically and be able to switch to appropriate modes, those are only applicable in light fog or a little rain. But in the case of downpour or dense fog, it is very difficult to get the objects which are hidden behind the raindrops and fog.

4. As the traffic sign standards are diverse, a robust natured classifier is required to work in all countries. Recognition is a multi-class problem as there are many signs to be recognized. An extendible and 
robust algorithm is necessary as different countries have a different set of signs and to handle various shapes and size. The neural network is one of the classic classifier used for the traffic sign recognition in this review. Random forest or SVM with handcrafted features like HOG, SURF or SIFT are other discussed algorithms in this review. From the review, it seems that an algorithm named deep convolutional neural network is being used vastly for TSDR which can extract and learn discriminative and robust features. Although an accurate selection of features and detection algorithms make an efficient TSDR, it requires high-end GPU and a large number of datasets. The large the datasets are the better it produces. The fact cannot be denied that it is hardly possible to pinpoint the best TSDR approach without comparing with other systems. The main cause for this is the lack of appropriate public traffic sign image dataset.

\section{DISCUSSION}

Current real-time road and traffic signs frameworks are far from the perfect state where it is insensitive to noise and brightness variations and had gone through a robust color segmentation process so that geometrical effects like scaling changes, translation, in-plane and out-plane have no adverse effects on the final result. Due to the continuously changing frame, it is quite difficult to produce a result that is both nearly accurate and has a low computational time. As observed above, it is apparent that tracking algorithms based on corners and edges extraction are exploited by motion blur. Even though some tracking algorithms have been introduced in cameras recently, but it lacks the robustness to exploit the minute details when the whole image is the scope. This makes Object tracking a challenging task when the whole image is used lessening the amount of relevant image information. On the other hand, producing a robust color segmentation with a good result for illumination variation is difficult as well and if night time traffic sign detection is taken into account then the lack of light cause collected images to be fuzzy, dark, low contrast with unclear details and limited dynamic range compression making detection even more difficult.

\section{CONCLUSION}

The main objective of this review is to analyze the key direction of the field of automatic traffic sign detection and recognition. In this review, the overview of the research in the field of TSDR system is provided with some current issues and challenges and the respond of the researchers are also given. In the following sections a comprehensive and a narrative study of the existing methods and frameworks. Current state-of-art, no humanmachine interactivity is observed which can be a part of future research as it is very necessary for an efficient Advanced Driving Assisting System. Another key problem is the availability of public database which is also needed to be solved. There are some databases that are publically available but still not widely used and only covered the Vienna Convention-Complaint. With highresolution camera and fast fps, it's necessary to develop a fast system. The illumination dependencies including nighttime detection can be solved by an adaptive color segmentation. Especially in night time color segmentation is most challenging as in sixteen-pixel camera the frames obtained produces too many noises. After color segmentation comes feature extraction in real time which becomes difficult due to the occurrence of motion blur. In real time solution motion blur is a common problem. But reducing motion blur also impacts the computational time. It is hard to maintain a balance between efficiency and accuracy. Also one of the objectives of this paper is to give a review on the detection and recognition the Bengali Traffic sign. Resources and datasets for Bengali traffic signs are still unavailable as of today. So research on Bengali traffic sign requires the collection of datasets from scratch. The overview of the research, the comprehensive study, and issues that are presented in the field of automatic TSDR in this paper with some future suggestion will hopefully lead to developing robust and efficient TSDR system because of its enormous range of applications.

\section{REFERENCES}

[1] P. Paclík, J. Novovičová, P. Pudil and P. Somol, "Road sign classification using Laplace kernel classifier", Pattern Recognition Letters, vol. 21, no. 13-14, pp. 11651173, 2000.

[2] M. Prieto and A. Allen, "Using self-organizing maps in the detection and recognition of road signs", Image and Vision Computing, vol. 27, no. 6, pp. 673-683, 2009.

[3] De la Escalera, J. Armingol and M. Mata, "Traffic sign recognition and analysis for intelligent vehicles", Image and Vision Computing, vol. 21, no. 3, pp. 247-258, 2003.

[4] M.Benallal and J. Meunier, "Real-time color segmentation of road signs", CCECE 2003 - Canadian Conference on Electrical and Computer Engineering. Toward a Caring and Humane Technology (Cat. No.03CH37436), vol. 3, pp.:1823-1826, 2003.

[5] M. M. Zadeh, T. Kasvand, and C. Y. Suen, "Localization and recognition of traffic signs for automated vehicle control systems", In Proc. SPIE Vol. 3207, Intelligent Transportation Systems, pages 272-282, 1998.

[6] A. de la Escalera, L. Moreno, M. Salichs and J. Armingol, "Road traffic sign detection and classification", IEEE Transactions on Industrial Electronics, vol. 44, no. 6, pp. 848-859, 1997.

[7] C. Bahlmann, Y. Zhu, Visvanathan Ramesh, M. Pellkofer and T. Koehler, "A system for traffic sign detection, tracking, and recognition using color, shape, and motion information", IEEE Proceedings. Intelligent Vehicles Symposium, 2005., pp. 255-260, 2005.

[8] S. Maldonado-Bascon, S. Lafuente-Arroyo, P. GilJimenez, H. Gomez-Moreno and F. Lopez-Ferreras, "Road-Sign Detection and Recognition Based on Support Vector Machines", IEEE Transactions on Intelligent Transportation Systems, vol. 8, no. 2, pp. 264-278, 2007.

[9] R. Malik, J. Khurshid and S. Ahmad, "Road Sign Detection and Recognition using Color Segmentation, 
Shape Analysis, and Template Matching", 2007 International Conference on Machine Learning and Cybernetics, vol. 6, pp. 3556-3560, 2007.

[10] H. Huang, C. Chen, Y. Jia and S. Tang, "Automatic Detection and Recognition of Circular Road Sign", 2008 IEEE/ASME International Conference on Mechatronic and Embedded Systems and Applications, pp. 626-630, 2008.

[11] P. Wanitchai and S. Phiphobmongkol, "Traffic Warning Signs Detection and Recognition Based on Fuzzy Logic and Chain Code Analysis", 2008 Second International Symposium on Intelligent Information Technology Application, pp. 508 - 512, 2008.

[12] K. C.G., L. Prabhu, A. V. and R. K., "Traffic Sign Detection and Pattern Recognition Using Support Vector Machine", 2009 Seventh International Conference on Advances in Pattern Recognition, pp. 87 - 90, 2009.

[13] W. Shadeed, D. Abu-Al-Nadi, and M. Mismar, "Road traffic sign detection in color images", 10th IEEE International Conference on Electronics, Circuits, and Systems, 2003. ICECS 2003. Proceedings of 2003, vol. 2, pp. 890 - 893, 2003.

[14] S. Vitabile, G. Pollaccia, G. Pilato and E. Sorbello, "Road signs recognition using a dynamic pixel aggregation technique in the HSV color space", Proceedings 11th International Conference on Image Analysis and Processing, pp. 572- 577, 2001.

[15] X. Gao, L. Podladchikova, D. Shaposhnikov, K. Hong and N. Shevtsova, "Recognition of traffic signs based on their color and shape features extracted using human vision models", Journal of Visual Communication and Image Representation, vol. 17, no. 4, pp. 675-685, 2006.

[16] P P. Paclík and J. Novovičová, "Road Sign Recognition without Color Information", in 6th Annual Conference of the Advances School for Computing and Imaging Conference, ASCI 2000, Belgium, 2000, pp. 84-90.

[17] G. Loy and N. Barnes, "Fast shape-based road sign detection for a driver assistance system," 2004 IEEE/RSJ International Conference on Intelligent Robots and Systems (IROS) (IEEE Cat. No.04CH37566), 2004, pp. 70-75 vol.1.

[18] S. Chakraborty and K. Deb, "Bangladeshi road sign detection based on YCbCr color model and DtBs vector," 2015 International Conference on Computer and Information Engineering (ICCIE), Rajshahi, 2015, pp. 158-161.

[19] Z. Malik and I. Siddiqi, "Detection and Recognition of Traffic Signs from Road Scene Images," 2014 12th International Conference on Frontiers of Information Technology, Islamabad, 2014, pp. 330-335.

[20] C. Liu, F. Chang, Z. Chen and D. Liu, "Fast Traffic Sign Recognition via High-Contrast Region Extraction and Extended Sparse Representation," in IEEE Transactions on Intelligent Transportation Systems, vol. 17, no. 1, pp. 79-92, Jan. 2016.

[21] S. Maldonado-Bascon, S. Lafuente-Arroyo, P. Siegmann, H. Gomez-Moreno and F. J. Acevedo-Rodriguez, "Traffic sign recognition system for inventory purposes," 2008 IEEE Intelligent Vehicles Symposium, Eindhoven, 2008, pp. 590-595.

[22] J. Greenhalgh and M. Mirmehdi, "Real-Time Detection and Recognition of Road Traffic Signs," in IEEE Transactions on Intelligent Transportation Systems, vol. 13, no. 4, pp. 1498-1506, Dec. 2012.

[23] S. Maldonado-Bascon, S. Lafuente-Arroyo, P. GilJimenez, H. Gomez-Moreno and F. Lopez-Ferreras, "Road-Sign Detection and Recognition Based on Support
Vector Machines," in IEEE Transactions on Intelligent Transportation Systems, vol. 8, no. 2, pp. 264-278, June 2007.

[24] Sin-Yu Chen and Jun-Wei Hsieh, "Boosted road sign detection and recognition," 2008 International Conference on Machine Learning and Cybernetics, Kunming, 2008, pp. 3823-3826.

[25] M. A. A. Sheikh, A. Kole and T. Maity, "Traffic sign detection and classification using color feature and neural network," 2016 International Conference on Intelligent Control Power and Instrumentation (ICICPI), Kolkata, 2016, pp. 307-311.

[26] Abedin, P. Dhar, M. K. Hossenand and K. Deb, "Traffic sign detection and recognition using fuzzy segmentation approach and artificial neural network classifier respectively," 2017 International Conference on Electrical, Computer and Communication Engineering (ECCE), Cox's Bazar, 2017, pp. 518-523.

[27] V. Ngoc Hanh and D. Park, "Traffic Sign Detection with Color Probability Map Using Artificial Neural Networks", International Journal of Applied Engineering Research, vol. 11, no. 21, pp. 10655-10658, 2016.

[28] Supreeth H. S. G and C. M. Patil, "An approach towards efficient detection and recognition of traffic signs in videos using neural networks," 2016 International Conference on Wireless Communications, Signal Processing and Networking (WiSPNET), Chennai, 2016, pp. 456-459.

[29] S. Jung, U. Lee, J. Jung and D. H. Shim, "Real-time Traffic Sign Recognition system with deep convolutional neural network," 2016 13th International Conference on Ubiquitous Robots and Ambient Intelligence (URAI), Xi'an, 2016, pp. 31-34.

[30] S. C. Huang, H. Y. Lin and C. C. Chang, "An in-car camera system for traffic sign detection and recognition," 2017 Joint 17th World Congress of International Fuzzy Systems Association and 9th International Conference on Soft Computing and Intelligent Systems (IFSA-SCIS), Otsu, 2017, pp. 1-6.

[31] P.Uršič, D. Tabernik, R. Mandeljc and D. Skočaj, "Towards large-scale traffic sign detection and recognition", in 22nd Computer Vision Winter Workshop, Retz, Austria, 2017.

[32] Z. Chen, X. Huang, Z. Ni and H. He, "A GPU-based realtime traffic sign detection and recognition system," 2014 IEEE Symposium on Computational Intelligence in Vehicles and Transportation Systems (CIVTS), Orlando, FL, 2014, pp.1-5.

[33] Y. Ma and L. Huang, "Hierarchical Traffic Sign Recognition Based on Multi-feature and Multi-classifier Fusion", Proceedings of the First International Conference on Information Science and Electronic Technology, 2015.

[34] A. Ellahyani, M. Ansari, I. Jaafari and S. Charfi, "Traffic Sign Detection and Recognition using Features Combination and Random Forests", International Journal of Advanced Computer Science and Applications, vol. 7, no. 1, pp. 686-693, 2016.

[35] K.Lim, H. Byun and Y. Choi, "A Real-time Traffic Sign Recognition System Based on Local Structure Features", in The 19th International Conference on Image Processing, Computer Vision, \& Pattern Recognition, 2015, pp. 65-68.

[36] J. H. Shi and H. Y. Lin, "A vision system for traffic sign detection and recognition," 2017 IEEE 26th International Symposium on Industrial Electronics (ISIE), Edinburgh, 2017, pp. 1596-1601. 
[37] F. Zaklouta and B. Stanciulescu, "Real-time traffic sign recognition in three stages", Robotics and Autonomous Systems, vol. 62, no. 1, pp. 16-24, 2014..

[38] H. Thanh, "Morphological Classification for Traffic Sign Recognition", Electrical and Electronic Engineering, vol. 4, no. 2, pp. 36-44., 2014.

[39] L. Chen, Q. Li, M. Li and Q. Mao, "Traffic sign detection and recognition for intelligent vehicle," 2011 IEEE Intelligent Vehicles Symposium (IV), Baden-Baden, 2011, pp. 908-913.

[40] H. N. Dean and K. V. Jabir, "Real Time Detection and Recognition of Indian Traffic Signs using Matlab", International Journal of Scientific \& Engineering Research, Volume 4, Issue 5, pp. 684-690, 2013.

[41] M. Kobayashi, M. Baba, K. Ohtani and L. Li, "A method for traffic sign detection and recognition based on genetic algorithm," 2015 IEEE/SICE International Symposium on System Integration (SII), Nagoya, 2015, pp. 455-460.

[42] X. Baro, S. Escalera, J. Vitria, O. Pujol and P. Radeva, "Traffic Sign Recognition Using Evolutionary Adaboost Detection and Forest-ECOC Classification," in IEEE Transactions on Intelligent Transportation Systems, vol. 10, no. 1, pp. 113-126, March 2009.

[43] M. Billah, S. Waheed, K. Ahmed and A. Hanifa, "Real Time Traffic Sign Detection and Recognition using Adaptive Neuro Fuzzy Inference System", Communications on Applied Electronics, vol. 3, no. 2, pp. $1-5,2015$.

[44] Soetedjo A., Yamada K. "Fast and robust traffic sign detection", Systems, man and cybernetics, vol. 2, pp. 1341-1346, 2005.

[45] Soetedjo A., Yamada K, "An efficient algorithm for traffic sign detection", Journal of advanced computational intelligence and intelligent informatics, vol. 10, no 3, pp. 409-418, 2005.

[46] S. Marsi et al., "Video Enhancement and Dynamic Range Control of HDR Sequences for Automotive Applications" EURASIP J. Advances in Signal Processing, vol. 2007, 2007, p. 9.

[47] M. García Garrido, M. Sotelo and E. Martm-Gorostiza, "Fast Traffic Sign Detection and Recognition Under Changing Lighting Conditions", in 2006 IEEE Intelligent Transportation Systems Conference, Toronto, Canada, 2006, pp. 811-816.

[48] W. J. Kuo and C. C. Lin, "Two-Stage Road Sign Detection and Recognition," 2007 IEEE International Conference on Multimedia and Expo, Beijing, 2007, pp. 1427-1430.

[49] S. Ren, K. He, R. B. Girshick, and J. Sun, "Faster R-CNN: Towards Real-Time Object Detection with Region Proposal Networks," in NIPS, 2015, pp. 91-99.

[50] E. Oruklu, D. Pesty, J. Neveux and J. E. Guebey, "Realtime traffic sign detection and recognition for in-car driver assistance systems," 2012 IEEE 55th International Midwest Symposium on Circuits and Systems (MWSCAS), Boise, ID, 2012, pp. 976-979.

[51] Z. Zheng, H. Zhang, B. Wang, and Z. Gao, "Robust traffic sign recognition and tracking for Advanced Driver Assistance. Systems," 15th International IEEE Conference on Intelligent Transportation Systems (ITSC), 2012, pp. 704-709.

[52] Z . Omary and F. Mtenzi, "Machine learning approach to identifying the dataset threshold for the performance estimators in supervised learning," International Journal for Infonomics (IJI), vol. 3, Sept. 2010, p:314-325.
[53] R. Michalski, J. Carbonell, and T. Mitchell, Machine Learning: An Artificial Intelligence Approach. Morgan Kaufmann, 1986.

[54] S. Vitabile, A. Gentile, and F. Sorbello, "A neural network based automatic road sign recognizer," presented at The 2002 Inter. Joint Conf. on Neural Networks, Honolulu, HI, USA, 2002.

[55] J. Miura, T. Kanda, and Y. Shirai, "An active vision system for real-time traffic sign recognition," presented at 2000 IEEE Intelligent Transportation Systems, Dearborn, MI, USA, 2000.

[56] S. Vitabile, A. Gentile, G. Dammone, and F. Sorbello, "Multi-layer perceptron mapping on a SIMD architecture," presented at The 2002 IEEE Signal Processing Society Workshop, 2002.

[57] Bangladesh Road Transport Authority, "BANGLADESH ROAD SIGN MANUAL", Bangladesh Road Transport Authority. Available: http://www.rhd.gov.bd/Documents/ConvDocs/Road\%20S ign\%20Manual\%20Volume-1.pdf [Accessed: 12- Dec2017]

[58] H. Cheng, X. Jiang, Y. Sun and J. Wang, "Color image segmentation: advances and prospects", Pattern Recognition, vol. 34, no. 12, pp. 2259-2281, 2001.

[59] H. Fleyeh, "Traffic and Road Sign Recognition", Ph.D., Napier University, 2008

[60] A. Ruta, Y. Li, and X. Liu, "Real-Time Traffic Sign Recognition from Video by Class-Specific Discriminative Features," Pattern Recognition, vol. 43, no. 1, 2010, pp. 416-30.

[61] "ADAC: BMW has best traffic-sign recognition", automotiveIT International, 2010 [Online]. Available: http://www.automotiveit.com/news/adac-bmw-has-besttraffic-sign-recognition/. [Accessed: 23- Jan- 2018]

[62] J. Levinson et al., "Towards fully autonomous driving: Systems and algorithms," 2011 IEEE Intelligent Vehicles Symposium (IV), Baden-Baden, 2011, pp. 163-168.

[63] J. Markoff, "Google Cars Drive Themselves, in Traffic", The New Work Times, 2010.

[64] Geonovum, "Self-Driving Vehicles \& Geo-Information", Geonovum [Online]. Available: https://www.geonovum.nl/sites/default/files/SelfDrivingVehiclesReport.pdf. [Accessed: 21- Feb- 2018]

[65] Michalski, Ryszard S., Carbonell, Jamie G. and Mitchell, Tom. M. Machine learning: An Artificial Intelligence Approach. Morgan Kaufmann, 1985.

[66] H. Bay, A. Ess, T. Tuytelaars, and L. V. Gool, "SURF: Speeded up robust features," Computer Vision and Image Understanding, vol. 110, no. 3, pp. 346-359, 2008

[67] D. Lowe, "Distinctive image features from scale-invariant key points, "International Journal of Computer Vision, vol. 60, no. 2, pp. 91-110, November 2004.

[68] L. Auria and R. Moro, "Support Vector Machines (SVM) as a Technique for Solvency Analysis", SSRN Electronic Journal, 2008.

[69] "Convolutional Neural Networks - Convolutional Neural Networks for Image and Video Processing - TUM Wiki", Wiki.tum.de, 2018. [Online]. Available: https://wiki.tum.de/display/lfdv/Convolutional+Neural+N etworks. [Accessed: 27- Feb- 2018]

[70] M. O. Rahman, F. A. Mousumi, E. Scavino, A. Hussain and $\mathrm{H}$. Basri, "Real time road sign recognition system using artificial neural networks for bengali textual information box," 2008 International Symposium on Information Technology, Kuala Lumpur, Malaysia, 2008, pp. 1-8 
[71] R. Gonzalez and R. Woods, Digital image processing. Upper Saddle River, N.J.: Prentice Hall, 2002.

[72] Y. Freund and R. E. Schapire, "Experiments with a new boosting algorithm," In International Conference on Machine Learning, 1996, pp. 148-156.

\section{Authors' Profiles}

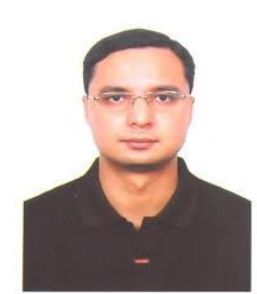

Dip Nandi working as a Senior Associate Professor in the Department of Computer Science at American International University Bangladesh. His research interests include E-Learning, Software Engineering, and Information Systems.

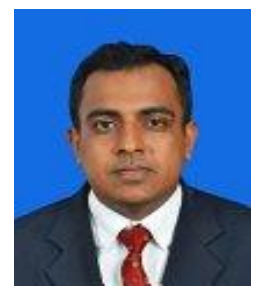

A.F.M. Saifuddin Saif received Ph.D. from Faculty of Information Science and Technology, University Kebangsaan Malaysia (UKM) in 2016. He received M.Sc. in Computer System Engineering (Software System) from University of East London, UK, and B.Sc. (Eng.) degree in Computer Science and Engineering from Shahjalal University of Science and Technology, Bangladesh in 2012 and 2008, respectively. Most of his contributions in Computer Vision and Artificial Intelligence Research field were published in ISI Q1 journals. He has published many papers in ISI indexed Journals, Scopus indexed Journals, Book Chapters, Conferences, and Proceedings. He served as Technical Committee Members, Reviewers, Guest Speakers, Session Chairs in many Conferences and Workshops. Currently, Dr. A.F.M. Saifuddin Saif is an Assistant Professor at Faculty of Information Science and Technology, American International University - Bangladesh. Before joining the university, he did Post Doctorate at Faculty of Information Science and Technology, University Kebangsaan Malaysia. He spent more than 6 years in IT industry such as Advanced Software Development, Web eMaze etc as IT researcher. His research interests include Image Processing, Computer Vision, Artificial Intelligence, Augmented Reality, 3D reconstruction, and Medical Image Processing.

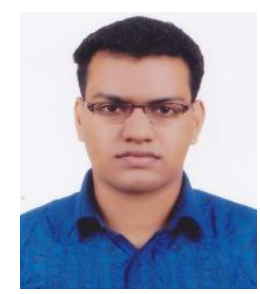

Prottoy Paul is an undergraduate student of Computer Science and Engineering under the Department of Science and Information Technology of American International University Bangladesh. His research interests and passion are mostly based on Computer Vision and Pattern Recognition, Image processing, Artificial Neural Network, Color Segmentation, Machine Learning, Object Tracking and Detection, Evolutionary Computation.

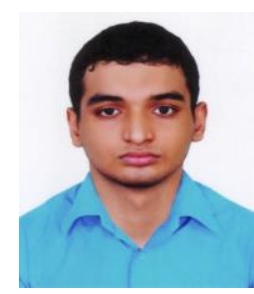

Kazi Md Zubair is an undergraduate student of Computer Science and Engineering under the Department of Science and Information Technology of American International University Bangladesh. His research interests and passion are mostly based on Convolutional Artificial Neural Networks, Object Detection and Tracking included in the field of Computer Vision, Image Processing and Machine Learning.

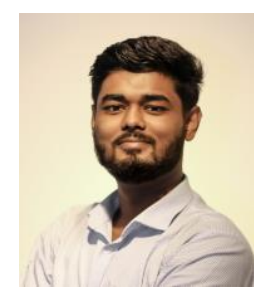

Seemanta Ahmed Shubho is a student of undergraduate (UG) program majoring in Computer Science \& Engineering from American International UniversityBangladesh. He is also working as an embedded software engineer for last two years. He has won Bracathon II in 2017 (hackathon organized by Brac). He was also a runner-up in the national hackathon 2016 organized by the Ministry of Information and Communication Technology. $\mathrm{He}$ has contributions in open source libraries. His research interest focuses but not limited to Image Processing, Computer Vision, Augmented Reality, Virtual Reality, Artificial Intelligence and Machine Learning.

How to cite this paper: Dip Nandi, A.F.M. Saifuddin Saif, Prottoy Paul, Kazi Md. Zubair, Seemanta Ahmed Shubho, " Traffic Sign Detection based on Color Segmentation of Obscure Image Candidates: A Comprehensive Study", International Journal of Modern Education and Computer Science(IJMECS), Vol.10, No.6, pp. 35-46, 2018.DOI: 10.5815/ijmecs.2018.06.05 